hep-th/0005068

HUTP-00/A013

\title{
Supergravity Instantons and the Universal Hypermultiplet
}

\author{
Michael Gutperle and Michał Spaliński \\ Jefferson Laboratory of Physics \\ Harvard University, Cambridge, MA 02138, USA \\ gutperle@riemann.harvard.edu, mspal@schwinger.harvard.edu
}

\begin{abstract}
The effective action of $N=2$ supersymmetric 5-dimensional supergravity arising from compactifications of M-theory on Calabi-Yau threefolds receives non-perturbative corrections from wrapped Euclidean membranes and fivebranes. These contributions can be interpreted as instanton corrections in the 5 dimensional field theory. Focusing on the universal hypermultiplet, a solution of this type is presented and the instanton action is calculated, generalizing previous results involving membrane instantons. The instanton action is not a sum of membrane and fivebrane contributions: it has the form reminiscent of non-threshold bound states.
\end{abstract}

May 2000 


\section{Introduction}

Nonperturbative effects in compactified string theories and M-theory can often be understood in terms of Euclidean wrapped branes. The study of such effects for M-theory and type II string theory was initiated by Becker, Becker and Strominger [1]. In the case of M-theory on a Calabi-Yau there are two types of effects: those arising from membranes wrapping special Lagrangian submanifolds and those from fivebranes wrapping the whole Calabi-Yau.

The simplest setting for a treatment of these effect is given by focusing exclusively on the universal hypermultiplet. This multiplet (leaving aside global issues [2]) is independent of the detailed structure of the Calabi-Yau. For M-theory and type IIA string theory compactified on rigid Calabi-Yau manifolds (with $h_{2,1}=0$ ) the universal hypermultiplet is the only hypermultiplet.

Hypermultiplets of $N=2$ supergravity in four and five dimensions parameterize quaternionic manifolds [3]. Classically the universal hypermultiplet lives in a $S U(2,1) / U(2)$ coset [4] [5]. Some aspects of perturbative corrections to the universal hypermultiplet obtained by dimensional reduction of higher derivative terms in M-theory were discussed in [6] [7][8]. On the other hand membrane and fivebrane instantons [1] will provide nonperturbative corrections to the metric on the moduli space of the hypermultiplets in the five dimensional supergravity. The study of such effects was continued in [9], which in particular investigated charge quantization and the breaking of continuous isometries of the quaternionic manifold due to instantons.

After compactification on a circle M-theory reduces to IIA superstring theory and the the M5 brane and M2 brane instanton will become a NS-5 brane and D2 brane instanton respectively. Instantons corresponding to wrapped D-branes can also be described using boundary states [10] and their effects can be analyzed [11] aanalogously to D-instantons in ten dimensional IIB theory [12]. 
Membrane instantons in compactifications with more supersymmetry were discussed [13] 14] 15] 18]. Membrane instantons in manifolds of exceptional holonomy (and $N=1$ supersymmetry) were discussed in [19] [20].

Solutions of ten dimensional IIB supergravity corresponding to D-instantons were first obtained in [21], see also [22] [23] [24]. These instantons carry "charges" associated with shifts of pseudoscalar fields. In the case of the universal hypermultiplet there are three pseudoscalars (in the four dimensional language they are the NS-NS axion and two RR-scalars).

In this paper the $N=2$ supergravity arising from dimensional reduction of eleven dimensional supergravity [25] on a Calabi-Yau threefold is studied with the intention of learning about nonperturbative corrections to the hypermultiplet moduli space arising from membranes and fivebranes wrapping Calabi-Yau cycles. Instanton solutions are found which carry all three of the charges which descend from membrane and fivebrane charges in 11 dimensions. By adding appropriate boundary terms to the Euclidean action the instanton action is evaluated. An interesting aspect of the result is that it is not a sum of membrane and fivebrane contributions, but has the form characteristic of a non-threshold bound state.

Section 2 reviews compactification of 11 dimensional supergravity and exhibits the quaternionic geometry and the isometries relevant to subsequent considerations. Section 3 discusses the charges. Supersymmetry preservation conditions are analyzed in section 4 . The instanton solution is presented in section 5. Section 6 is devoted to the evaluation of the instanton action. Section 7 describes the broken and unbroken supersymmetries and offers some remarks on computing the fermion determinant. Conclusions and a discussion of the results is given in section 8 . 


\section{Eleven dimensional supergravity on a Calabi-Yau manifold}

This section reviews the reduction of eleven dimensional supergravity on a CalabiYau threefold. The bosonic part of the action of eleven dimensional supergravity [25] is given by

$$
S=\frac{1}{2 k_{11}^{2}} \int d^{11} x \sqrt{-g}\left(R-\frac{1}{48} F^{M N P Q} F_{M N P Q}\right)-\frac{1}{12 k_{11}^{2}} \int A \wedge F \wedge F .
$$

The supersymmetry transformation of the gravitino in eleven dimensional supergravity is

$$
\delta_{\epsilon} \psi_{M}=\partial_{M} \epsilon+\frac{1}{4} \omega \frac{A B}{M} \Gamma_{\underline{A B}} \epsilon-\frac{1}{288}\left(\Gamma_{M}^{N P Q R}-8 \delta_{M}^{N} \Gamma^{P Q R}\right) \epsilon F_{N P Q R} .
$$

The notation here is that $\underline{A}, \underline{B}$ denote tangent space indices and $M, N$ denote world indices.

Dimensional reduction of eleven dimensional supergravity on a Calabi-Yau manifold (with vanishing G-fluxes), produces five dimensional $N=2$ supergravity with $h_{1,1}-1$ vectormultiplets and $h_{2,1}+1$ hypermultiplets [26]. The rest of the paper will focus on the dynamics of the universal hypermultiplet, which is present in any Calabi-Yau compactification.

The coordinates are split according to $x^{M}=\left(x^{\mu}, y^{m}\right)$, with $x^{\mu}, \mu=1, \cdots, 6$ parameterizing the internal Calabi-Yau space and $y^{m}, m=0, \cdots, 4$ parameterizing the transverse space. The ansatz for the metric is

$$
d s^{2}=e^{-1 / 3 \sigma\left(y^{2}\right)} d s_{C Y}^{2}+e^{2 / 3 \sigma\left(y^{2}\right)}\left(d y_{m}\right)^{2},
$$

where $d s_{C Y}^{2}$ is an unspecified Ricci flat metric of the Calabi-Yau manifold and $y^{2} \equiv y^{m} y^{m}$. The other fields in the universal hypermultiplet are the three form potential $C_{m n k}$ (which is dual to a scalar) and two real scalar fields $\chi_{1}, \chi_{2}$ defined by

$$
C_{\alpha \beta \gamma}=\frac{1}{\sqrt{2}} \chi \Omega_{\alpha \beta \gamma}, \quad C_{\bar{\alpha} \bar{\beta} \bar{\gamma}}=\frac{1}{\sqrt{2}} \bar{\chi} \bar{\Omega}_{\bar{\alpha} \bar{\beta} \bar{\gamma}},
$$

where $\chi=\chi_{1}+i \chi_{2}$ and $\bar{\chi}=\chi_{1}-i \chi_{2}$. Here $\Omega, \bar{\Omega}$ are the unique harmonic $(3,0)$ and $(0,3)$ forms on the Calabi-Yau manifold. 
Using (3) and (4), the bosonic part of the action for the universal hypermultiplet is given by

$$
\begin{aligned}
S & =-\frac{1}{2 \kappa_{5}^{2}} \int d^{5} x \sqrt{g}\left(\partial_{m} \sigma \partial_{m} \sigma+\frac{1}{24} e^{-2 \sigma} F_{m n p q} F^{m n p q}+e^{\sigma} \partial_{m} \chi \partial_{m} \bar{\chi}\right) \\
& +\frac{i}{48 \kappa_{5}^{2}} \int d^{5} x \epsilon^{m n p q l} F_{m n p q}\left(\bar{\chi} \partial_{l} \chi-\chi \partial_{l} \bar{\chi}\right) .
\end{aligned}
$$

In the following we set the five dimensional Newton constant $\kappa_{5}=1$ for notational convenience. In order to exhibit the quaternionic structure of the hypermultiplet moduli space the four form field strength has to be dualized. This is accomplished by introducing a Lagrange multiplier field $D$ and modifying the action as follows:

$$
S^{\prime}=S-\frac{1}{24} \int d^{5} x \epsilon^{m n p q l} F_{m n p q} \partial_{l} D
$$

Integrating out $D$ enforces the Bianchi identity $d F=0$ and one recovers the original action (5). If, on the other hand, one integrates out $F$ the dual description is obtained. The equation of motion for $F$ is given by

$$
e^{-2 \sigma} F^{m n p q}+\frac{i}{4} \epsilon^{m n p q l}\left(\bar{\chi} \partial_{l} \chi-\chi \partial_{l} \bar{\chi}\right)-\epsilon^{m n p q l} \partial_{l} D=0
$$

Using this relation the dual action becomes

$$
S^{\prime}=-\int d^{5} x\left(\frac{1}{2} \partial_{m} \sigma \partial_{m} \sigma+\frac{1}{2} e^{\sigma} \partial_{m} \chi \partial_{m} \bar{\chi}+\frac{1}{2} e^{2 \sigma}\left(\partial_{l} D-\frac{i}{4}\left(\bar{\chi} \partial_{l} \chi-\chi \partial_{l} \bar{\chi}\right)\right)^{2}\right) .
$$

In this dualized form the four scalars in the universal hypermultiplet parameterize the quaternionic manifold $S U(2,1) / U(2)$ [5] [6]. There are three isometries of this space, which are associated with shifts of the axionic fields $\chi, \bar{\chi}$ and $D[5][6][9]$ :

$$
\chi \rightarrow \chi+\epsilon, \quad \bar{\chi} \rightarrow \bar{\chi}+\bar{\epsilon}, \quad D \rightarrow D+\delta+\frac{i}{4}(\chi \bar{\epsilon}-\bar{\chi} \epsilon)
$$

with constant parameters $\epsilon=\epsilon_{1}+i \epsilon_{2}$ and $\delta$. These isometries are broken to a discrete subgroup by instanton effects [9]. 


\section{Charges}

Solutions of the supergravity equations of motion may carry charges which descend from the fivebrane and membrane charges of M-theory. The solutions of interest here are characterized by three charges associated with shifts of $D, \chi$ and $\bar{\chi}$ : these correspond to fivebranes wrapped on the entire Calabi-Yau manifold, and to membranes wrapping 3-cycles in the Calabi-Yau manifold respectively. The topological charge associated with the fivebrane is given by

$$
Q_{5}=\oint_{S_{\infty}^{4}} F .
$$

The two membrane charges are the Noether charges associated with shifts of $\chi$ and $\bar{\chi}$ :

$$
\begin{aligned}
& Q_{2}=\oint_{S_{\infty}^{4}}\left(e^{\sigma} * d \chi-i \chi F\right)=q_{2}+i Q_{5} \chi_{\infty} \\
& \bar{Q}_{2}=\oint_{S_{\infty}^{4}}\left(e^{\sigma} * d \bar{\chi}+i \bar{\chi} F\right)=\bar{q}_{2}-i Q_{5} \bar{\chi}_{\infty}
\end{aligned}
$$

where $\chi_{\infty}$ and $\bar{\chi}_{\infty}$ are the asymptotic values (at $r=\infty$ ) of $\chi$ and $\bar{\chi}$ respectively. Unlike the Noether charges $Q_{2}, \bar{Q}_{2}$ the charges $q_{2}, \bar{q}_{2}$ are invariant under shifts of $\chi_{\infty}$ and $\bar{\chi}_{\infty}$. This behavior can be traced to the presence of the Chern-Simons term in the eleven dimensional supergravity action (11).

\section{Supersymmetry}

The focus of interest here are instanton solutions which preserve four of the eight supersymmetries of the $d=5 N=2$ supergravity. The supersymmetry transformations of the five dimensional fields can easily be derived from the eleven dimensional ones (2) (see e.g. [27]). The hyperino transformations are given by

$$
\begin{aligned}
\delta \xi^{1} & =\frac{1}{3}\left(\partial_{n} \sigma \gamma^{n}-\frac{i}{24} e^{-\sigma} \epsilon^{m n p q r} F_{m n p q} \gamma_{r}\right) \epsilon^{1}+\frac{1}{3} e^{\sigma / 2} \partial_{n} \chi \gamma^{n} \epsilon^{2} \\
\delta \xi^{2} & =-\frac{1}{3} e^{\sigma / 2} \partial_{n} \bar{\chi} \gamma^{n} \epsilon^{1}+\frac{1}{3}\left(\partial_{n} \sigma \gamma^{n}+\frac{i}{24} e^{-\sigma} \epsilon^{m n p q r} F_{m n p q} \gamma_{r}\right) \epsilon^{2}
\end{aligned}
$$


These transformations can be succinctly expressed in terms of a two-by-two matrix $M$ (with gamma matrix entries) as $\delta \xi^{i}=M_{j}^{i} \epsilon^{j}$. Supersymmetry transformations for the gravitini are given by

$$
\begin{aligned}
& \delta \psi_{m}^{1}=\left(\nabla_{m}+\frac{i}{288} e^{-\sigma} \epsilon_{m}^{n p q r} F_{n p q r}\right) \epsilon_{1}-\frac{1}{3} e^{\sigma / 2} \partial_{m} \chi \epsilon_{2} \\
& \delta \psi_{m}^{2}=\frac{1}{3} e^{\sigma / 2} \partial_{m} \bar{\chi} \epsilon_{1}+\left(\nabla_{m}-\frac{i}{288} e^{-\sigma} \epsilon_{m}^{n p q r} F_{n p q r}\right) \epsilon_{2}
\end{aligned}
$$

Supersymmetry transformations for the dualized action (8) are given by eliminating $F$ in (12) (13) using the relation (7). They then take on the standard form for $N=2$ hypermultiplets [3].

\section{Instanton solution}

An instanton in a supergravity theory is a solution to the Euclidean equations of motion which is localized in the five dimensional (Euclidean) space time. It carries charges associated with shift symmetries of (axionic) scalars. There is an important sublety concerning the question how these axionic scalars are treated when the action is continued from Minkowski to Euclidean signature. The point of view assumed here is that one multiplies the (real) pseudoscalar fields by a factor of $i$ (this changes the sign of the kinetic term for $\chi, \bar{\chi})$. Note that this prescription seemingly makes the real part of the instanton action unbounded from below; this is however rectified by the boundary term introduced in section 6 团.

The action (5) then becomes

$$
\begin{aligned}
S_{\text {eucl }} & =\int d^{5} x \sqrt{g}\left(\frac{1}{2} \partial_{m} \sigma \partial_{m} \sigma+\frac{1}{48} e^{-2 \sigma} F_{m n p q} F^{m n p q}-\frac{1}{2} e^{\sigma} \partial_{m} \chi \partial_{m} \bar{\chi}\right) \\
& -\frac{1}{96} \int d^{5} x \epsilon^{m n p q l} F_{m n p q}\left(\bar{\chi} \partial_{l} \chi-\chi \partial_{l} \bar{\chi}\right) .
\end{aligned}
$$

An important simplifying assumption is that the solution is taken to be rotationally symmetric in Euclidean spacetime, i.e. all fields are assumed to depend only on $r \equiv\left|y-y_{0}\right|$, where $y_{0}$ is the location of the instanton.

1 See [28] 29] 24] 20] for a detailed discussion of this issue. 
The required solution is characterized by three charges associated with shifts of $D$, $\chi$ and $\bar{\chi}$ (as discussed in section 3): these correspond to fivebranes wrapped on the entire Calabi-Yau manifold, and to membranes wrapping 3-cycles in the Calabi-Yau manifold.

The instanton solution should be BPS, i.e. it should preserve half of the eight supersymmetries parameterized by two spinors $\epsilon_{1}, \epsilon_{2}$. For rotationally invariant field configurations the condition that $\delta \xi_{i}=0$ in (12) can be reduced to a two by two matrix equation. The BPS condition is then equivalent to the condition that this matrix has a zero eigenvalue. This implies,

$$
\partial_{m} \sigma \partial_{m} \sigma-\frac{1}{24} e^{-2 \sigma} F_{m n p q} F^{m n p q}-e^{\sigma} \partial_{m} \chi \partial_{m} \bar{\chi}=0 .
$$

The Ansatz for the metric is particularly simple: in the Einstein frame, the metric is flat $g_{m n}=\delta_{m n}$. In addition the following Ansatz for the 4-form field strength is made:

$$
F_{m n p q}=\epsilon_{m n p q r} \partial_{r} H
$$

The Bianchi identity for $F$ then implies that $H$ is harmonic, hence

$$
H=\frac{1}{8 \pi^{2}} \frac{Q_{5}}{r^{3}} .
$$

The normalization here has been chosen so that the parameter $Q_{5}$ appearing above is the fivebrane charge, as defined in the previous section.

Using (16) the equation of motion for $\sigma$ derived from the action (14) is given by

$$
\partial_{m} \partial_{m} \sigma+\frac{1}{2} e^{\sigma} \partial_{m} \chi \partial_{m} \bar{\chi}+e^{-2 \sigma} \partial_{m} H \partial_{m} H=0,
$$

and the BPS condition (15) becomes

$$
\partial_{m} \sigma \partial_{m} \sigma-e^{\sigma} \partial_{m} \chi \partial_{m} \bar{\chi}-e^{-2 \sigma} \partial_{m} H \partial_{m} H=0 .
$$

These equations simplify due to the assumed symmetry. By combining (15) and (18) $\chi, \bar{\chi}$ can be eliminated and one obtains an ordinary differential equation for $\sigma$ alone:

$$
\sigma^{\prime \prime}+\frac{4}{r} \sigma^{\prime}+\frac{1}{2}\left(\sigma^{\prime}\right)^{2}+\frac{9}{2} \frac{Q_{5}^{2}}{\left(8 \pi^{2}\right)^{2}} \frac{1}{r^{8}} e^{-2 \sigma}=0,
$$


where the prime denotes differentiation with respect to $r$. It is straightfoward to check that

$$
\sigma(r)=\sigma_{\infty}+\ln \left(1+\frac{b_{1}}{r^{3}}\right)+\ln \left(1+\frac{b_{2}}{r^{3}}\right)
$$

is the most general solution of (20), provided that the following relation between the parameters $\sigma_{\infty}, b_{1}, b_{2}$ and $Q_{5}$ holds:

$$
\left(b_{1}-b_{2}\right)^{2}-\frac{Q_{5}^{2}}{\left(8 \pi^{2}\right)^{2}} e^{-2 \sigma_{\infty}}=0 \text {. }
$$

The equations of motion for $\chi$ and $\bar{\chi}$ following from variations of (14) which vanish at the boundary 2 read

$$
\begin{aligned}
& \partial_{n}\left(e^{\sigma} \partial_{n} \chi-\partial_{n} H \chi\right)=0 \\
& \partial_{n}\left(e^{\sigma} \partial_{n} \bar{\chi}+\partial_{n} H \bar{\chi}\right)=0 .
\end{aligned}
$$

Due to radial symmetry these equations can be integrated once and one gets two first order differential equations

$$
\begin{aligned}
& e^{\sigma} \chi^{\prime}+\frac{Q_{5}}{8 \pi^{2}} \frac{1}{r^{4}} \chi=\frac{\alpha Q_{5}}{8 \pi^{2}} \frac{1}{r^{4}} \\
& e^{\sigma} \bar{\chi}^{\prime}-\frac{Q_{5}}{8 \pi^{2}} \frac{1}{r^{4}} \bar{\chi}=\frac{\bar{\alpha} Q_{5}}{8 \pi^{2}} \frac{1}{r^{4}}
\end{aligned}
$$

where $\alpha, \bar{\alpha}$ are integration constants. Note that the charges (11) are related to the integration constants in (24) by $Q_{2}=\alpha Q_{5}, \bar{Q}_{2}=\bar{\alpha} Q_{5}$.

These equations are easily integrated, and the result is

$$
\begin{aligned}
& \chi(r)=\frac{q_{2}}{Q_{5}}\left(\frac{1+b_{1} / r^{3}}{1+b_{2} / r^{3}}\right)+\alpha, \\
& \bar{\chi}(r)=-\frac{\bar{q}_{2}}{Q_{5}}\left(\frac{1+b_{2} / r^{3}}{1+b_{1} / r^{3}}\right)+\bar{\alpha} .
\end{aligned}
$$

The integration constants $\alpha, \bar{\alpha}$ can be related to the asymptotic values of the fields $\chi, \bar{\chi}$ at infinity:

$$
\begin{aligned}
& \chi_{\infty}=\alpha+\frac{q_{2}}{Q_{5}}, \\
& \bar{\chi}_{\infty}=\bar{\alpha}-\frac{\bar{q}_{2}}{Q_{5}} .
\end{aligned}
$$

2 Because some fields are singluar at $x=x_{0}$, one has to exclude an infinitesimal sphere around $x_{0}$, in addition to the boundary at infinity. 
So far only one linear combination of equations (15) and (18) has been used. Requiring that these are both satisfied in addition to (22) imposes a second condition on the parameters:

$$
\left(b_{1}+b_{2}\right)^{2}-\frac{Q_{5}^{2}}{\left(8 \pi^{2}\right)^{2}} e^{-2 \sigma_{\infty}}-\frac{q_{2} \bar{q}_{2}}{\left(8 \pi^{2}\right)^{2}} e^{-\sigma_{\infty}}=0 .
$$

The two relations (22) and (27) determine the parameters $b_{1}, b_{2}$ in (21) in terms of the asymptotic value of $\sigma$ and the charges,

$$
\begin{aligned}
& b_{1}=\frac{1}{16 \pi^{2}}\left(\sqrt{Q_{5}^{2} e^{-2 \sigma_{\infty}}+q_{2} \bar{q}_{2} e^{-\sigma_{\infty}}}+Q_{5} e^{-\sigma_{\infty}}\right), \\
& b_{2}=\frac{1}{16 \pi^{2}}\left(\sqrt{Q_{5}^{2} e^{-2 \sigma_{\infty}}+q_{2} \bar{q}_{2} e^{-\sigma_{\infty}}}-Q_{5} e^{-\sigma_{\infty}}\right) .
\end{aligned}
$$

Exchanging $b_{1}$ and $b_{2}$ in the solution, corresponds to exchanging $\chi_{1}$ and $\chi_{2}$. In the following section it is shown that the instanton action is independent of the asymptotic values of $\chi$, $\bar{\chi}$ at infinity.

Note that choosing the five dimensional metric to be flat is consistent, since the stress tensor derived from (14) vanishes on shell.

In general the instanton solution constructed above carries three charges $Q_{5}, q_{2}, \bar{q}_{2}$ and naturally generalizes the solutions carrying only a single charge given in [1] [9] [22]. The Euclidean instanton solutions have an interpretation as (complex) saddle points of the Euclidean action which describe tunneling between vacua with different charges. Note that away from asymptotic infinity the solution becomes complex, since (25) implies that $\chi$ is the complex conjugate of $\bar{\chi}$ only in the limit $r \rightarrow \infty$.

\section{The instanton action}

The Euclidean action (14) is not invariant under constant shifts of $\chi, \bar{\chi}$. The invariance under such shifts can be restored by adding a total derivative term to the action (14):

$$
S_{\text {eucl }}^{\prime}=S_{\text {eucl }}+\frac{1}{4} \int d^{5} x \sqrt{g} \nabla^{n}\left(e^{\sigma}\left(\nabla_{n} \chi \bar{\chi}+\nabla_{n} \bar{\chi} \chi\right)\right)
$$


Under constant shifts of $\chi, \bar{\chi}$ the action $S^{\prime}$ transforms into itself up to a term proportional to the equation of motion.

Adding a total derivative term is equivalent to adding a boundary term to the action and this does not change the equations of motion. The boundary term does however contribute to the instanton action. Using the solutions of the equations of motion in the action (29) gives

$$
S_{\text {inst }}=-\oint \partial_{n} \sigma
$$

(the equation of motion (19) was used to express the action as a total derivative). Evaluating (30) using the solution for $\sigma$ given in (21) together with (28) leads to

$$
S_{\text {inst }}=8 \pi^{2}\left(b_{1}+b_{2}\right)=\sqrt{Q_{5}^{2} e^{-2 \sigma_{\infty}}+q_{2} \bar{q}_{2} e^{-\sigma_{\infty}}} .
$$

Note that, as expected, the boundary term in (29) removes the dependence of the instanton action on $\alpha, \bar{\alpha}$ (or equivalently on $\chi_{\infty}, \bar{\chi}_{\infty}$ ). The weight with which an instanton contributes in physical processes is given by $\exp \left(-S_{\text {inst }}\right)$. Just as in the case of the $\theta$ term in the action for Yang-Mills instantons the only dependence on the asympotic value of the axionic scalars $D, \chi, \bar{\chi}$ is given by a phase factor $\exp (i \theta)$

$$
\exp (i \theta)=\exp \left(i\left(Q_{5} D_{\infty}+\frac{1}{4} Q_{2} \bar{\chi}_{\infty}+\frac{1}{4} \bar{Q}_{2} \chi_{\infty}\right)\right)
$$

This term will imply that only discrete shifts of the axionic scalars are a symmetry when instantons are taken into account $[9]$. In the path integral this term has the role of a boundary term imposes fixed boundary conditions on the charges [28] [29].

This form of the instanton action generalizes the instanton actions for a single charge.

When two of the three charges $Q_{5}, \operatorname{Re}\left(q_{2}\right), \operatorname{Im}\left(q_{2}\right)$ are set to zero it reduces to the familiar expression for the fivebrane and membrane instanton actions.

3 The discussion of the phase is not complete without the investigation of the one loop determinants, but this lies beyond the scope of our paper. 
Reduction of the five dimensional theory on a circle relates M-theory compactified on a Calabi-Yau manifold to Type IIA string theory compactified on the same CalabiYau. The five-dimensional universal hypermultiplet turns into the four dimensional one. The Calabi-Yau volume (breathing mode) $\sigma$ is mapped to the four dimensional dilaton by $\sigma=2 \phi_{I I A}$ [6] [9]. The four dimensional instanton action is then obtained by this replacement. The resulting dependence on the string coupling $g=e^{\phi_{I I A}}$ is what is expected for NS-fivebrane and D2 brane instantons respectively.

\section{Broken and unbroken supersymmetries}

The explicit form of the unbroken supersymmetries can be determined from the conditions (12),(13). Substituting the condition $\delta \xi^{i}=0$ into $\delta \psi^{i}=0$ gives the following equations for the supersymmetry transformation parameters $\epsilon^{i}$ :

$$
\begin{aligned}
& \left(\nabla_{r}+\frac{1}{3} \partial_{r} \sigma+\frac{1}{4} e^{-\sigma} \partial_{r} H\right) \epsilon^{1}=0 \\
& \left(\nabla_{r}+\frac{1}{3} \partial_{r} \sigma-\frac{1}{4} e^{-\sigma} \partial_{r} H\right) \epsilon^{2}=0 .
\end{aligned}
$$

Using the explicit form of $\sigma$ given in (21) and $H$ given in (17) one finds

$$
\begin{aligned}
& \epsilon^{1}(r)=\left(1+\frac{b_{1}}{r^{3}}\right)^{-7 / 12}\left(1+\frac{b_{2}}{r^{3}}\right)^{-1 / 12} \hat{\epsilon}^{1}, \\
& \epsilon^{2}(r)=\left(1+\frac{b_{2}}{r^{3}}\right)^{-7 / 12}\left(1+\frac{b_{1}}{r^{3}}\right)^{-1 / 12} \hat{\epsilon}^{2},
\end{aligned}
$$

where $\hat{\epsilon}_{1}, \hat{\epsilon}_{2}$ are constant spinors. The four unbroken supersymmetries are then found by demanding that $\delta \xi_{i}=0$. Using (34) this condition reduces to a matrix equation for the $\hat{\epsilon}_{i}$.

$$
\left(\begin{array}{cc}
b_{1}+b_{2}+e^{-\sigma_{\infty} \frac{Q_{5}}{8 \pi^{2}}} & i e^{-\sigma_{\infty} / 2 \frac{q_{2}}{8 \pi^{2}}} \\
-i e^{-\sigma_{\infty} / 2} \frac{Q_{2}}{8 \pi^{2}} & b_{1}+b_{2}-e^{-\sigma_{\infty} \frac{Q_{5}}{8 \pi^{2}}}
\end{array}\right)\left(\begin{array}{c}
\hat{\epsilon}^{1} \\
\hat{\epsilon}^{2}
\end{array}\right)=0
$$

Note that the condition (27) is the same as the BPS condition that the determinant of the matrix (35) vanishes.

It is well known that the broken supersymmetries in the instanton background will generate fermionic zero modes. Scattering amplitudes vanish in the instanton background 
unless the fermionic zero modes are absorbed by field insertions. This leads to new instanton induced interactions. The simplest of such interactions are four fermion terms [1] [9], which involve the Riemann tensor of the quaternionic moduli space. The presence of such terms presumably implies that the metric on the quaternionic moduli also receives instanton corrections.

The explicit form of the four-fermion terms will not be discussed here, but it is easy to see that the fermionic zero modes will be normalizable. As defined in (12) the hyperinos transform as $\delta \xi^{i}=M_{j}^{i} \epsilon^{j}$. In the instanton background one has $\operatorname{det}(M)=0$, whereas the trace of the matrix $M$ is given by $2 / 3 \gamma^{r} \partial_{r} \sigma$. Hence the broken supersymmetries will correspond to the eigenvector with eigenvalue $2 / 3 \partial_{r} \sigma$. The norm of the broken supersymmetry $|\delta \xi|^{2}$ will therefore contain

$$
\partial_{r} \sigma \partial_{r} \sigma=\frac{1}{r^{8}} \frac{\left(b_{1}+b_{2}+2 b_{1} b_{2} / r^{3}\right)^{2}}{\left(1+b_{1} / r^{3}\right)^{2}\left(1+b_{2} / r^{3}\right)^{2}}
$$

This expression behaves like $1 / r^{8}$ as $r \rightarrow \infty$ and like $1 / r^{2}$ as $r \rightarrow 0$. Hence $|\delta \xi|^{2} /|\epsilon|^{2}$ has a finite integral over $R^{5}$ and the broken supersymmetries will be normalizable. In principle one can use the explicit form of the solution to calculate the form of the instanton induced terms.

\section{Discussion}

This paper presented a solution of the Euclidean equations of motion for the universal hypermultiplet in five dimensional $N=2$ supergravity, which generalizes solutions which carry only one charge (and reduces to them in the limit when two of the three charges are set to zero). The action of the instanton has interesting properties. The action of a multiple charge instanton is not the sum of the actions of instantons carrying a single charge: its form is characteristic of a "non threshold bound state". This means that the instantons cannot be separated: a configuration of single-charge instantons at different spacetime points would not be supersymmetric. Since the fivebrane and two brane solutions preserve different supersymmetries this has to be the case. In [31] 32] a solution of 
eleven dimensional supergravity in flat space was found which can be interpreted as a non threshold bound state of a M2 brane within a M5 brane. In [33] it was shown that the tensions behave exactly as in (31).

It would be very interesting to understand the geometric conditions for the supersymmetric wrapping of a Euclidean M5 brane on a Calabi-Yau which follow from the existence of BPS instantons in analogy with the analysis in [1]. Presumably one would need to understand the Euclidean fivebrane worldvolume theory [34]35] in the presence

of three form fluxes [36] [37]. Since the worldvolume action of M5 branes is considerably more complicated than the one of M2 branes this is not straightforward (see however [38], where the M5 brane worldvolume theory is related to Kodaira-Spencer theory). The use of calibrations might also prove useful in this respect.

The classical saddle point of the Euclidean action discussed here is only the starting point of an instanton calculation. In particular, integration over fermionic zero modes and the evaluation of fluctuation determinants would have to be performed to obtain an explicit form of the corrections to the hypermultiplet moduli space metric. Fermionic zero modes can be analyzed to some extent within the framework of supergravity, as briefly indicated in this note. However, as discussed in [19], the rules for calculating the fluctuation determinants are not properly understood M-theory, unlike in field theories where a second quantized path integral is at our disposal. This is obviously an important open problem. As is sometimes the case with D-instantons, the best hope might be to use heterotic - type II duality, which relates spacetime instantons of type II string theory to world sheet instantons on the heterotic side, to learn something about instanton calculus for $N=2$ backgrounds.

\section{Acknowledgements}

We are grateful to A. Strominger and V. Balasubramanian for useful conversations. The work of M.G. is supported in part by the David and Lucile Packard Foundation. The work of M.S. was supported in part by NSF grant PHY-98-02709. 


\section{References}

[1] K. Becker, M. Becker and A. Strominger, "Five-branes, membranes and nonperturbative string theory," Nucl. Phys. B456 (1995) 130 hep-th/9507158].

[2] P. S. Aspinwall, "Compactification, geometry and duality: N = 2," hep-th/0001001.

[3] J. Bagger and E. Witten, "Matter Couplings In N=2 Supergravity ," Nucl. Phys. B222 (1983) 1.

[4] S. Cecotti, S. Ferrara and L. Girardello, "Geometry Of Type II Superstrings And The Moduli Of Superconformal Field Theories," Int. J. Mod. Phys. A4 (1989) 2475.

[5] S. Ferrara and S. Sabharwal, "Quaternionic Manifolds For Type II Superstring Vacua Of Calabi-Yau Spaces," Nucl. Phys. B332 (1990) 317.

[6] A. Strominger, "Loop corrections to the universal hypermultiplet," Phys. Lett. B421 (1998) 139 hep-th/9706195.

[7] I. Antoniadis, S. Ferrara, R. Minasian and K. S. Narain, " ${ }^{* *} 4$ couplings in M- and type II theories on Calabi-Yau spaces," Nucl. Phys. B507 (1997) 571

[8] H. Gunther, C. Herrmann and J. Louis, "Quantum corrections in the hypermultiplet moduli space," Fortsch. Phys. 48 (2000) 119 hep-th/9901137.

[9] K. Becker and M. Becker, "Instanton action for type II hypermultiplets," Nucl. Phys. B551 (1999) 102 hep-th/9901126].

[10] H. Ooguri, Y. Oz and Z. Yin, "D-branes on Calabi-Yau spaces and their mirrors," Nucl. Phys. B477 (1996) 407 [hep-th/9606112].

[11] M. Gutperle and Y. Satoh, "D-branes in Gepner models and supersymmetry," Nucl. Phys. B543 (1999) 73 [hep-th/9808080].

[12] M. B. Green and M. Gutperle, "Effects of D-instantons," Nucl. Phys. B498 (1997) 195 hep-th/9701093.

[13] J. A. Harvey and G. Moore, "Fivebrane instantons and $\mathrm{R}^{* *} 2$ couplings in $\mathrm{N}=4$ string theory," Phys. Rev. D57 (1998) 2323 hep-th/9610237.

[14] C. Bachas, C. Fabre, E. Kiritsis, N. A. Obers and P. Vanhove, "Heterotic/type-I duality and D-brane instantons," Nucl. Phys. B509 (1998) 33 hep-th/9707126.

[15] B. Pioline and E. Kiritsis, "U-duality and D-brane combinatorics," Phys. Lett. B418 (1998) 61 hep-th/9710078.

[16] E. Kiritsis, N. A. Obers and B. Pioline, "Heterotic/type II triality and instantons on K3," JHEP 0001 (2000) 029 hep-th/0001083.

[17] A. B. Hammou and J. F. Morales, "Fivebrane instantons and higher derivative couplings in type I theory," hep-th/9910144.

[18] W. Lerche and S. Stieberger, "1/4 BPS states and non-perturbative couplings in $\mathrm{N}=$ 4 string theories," hep-th/9907133.

[19] J. A. Harvey and G. Moore, "Superpotentials and membrane instantons," hepth/9907026. 
[20] B. S. Acharya, "M theory, Joyce orbifolds and super Yang-Mills," ATMP 3 (1999) 227 hep-th/9812205.

[21] G. W. Gibbons, M. B. Green and M. J. Perry, "Instantons and Seven-Branes in Type IIB Superstring Theory," Phys. Lett. B370, 37 (1996) [hep-th/9511080].

[22] K. Behrndt, I. Gaida, D. Lust, S. Mahapatra and T. Mohaupt, "From type IIA black holes to T-dual type IIB D-instantons in $\mathrm{N}=2, \mathrm{D}=4$ supergravity," Nucl. Phys. B508 (1997) 659 [hep-th/9706096].

[23] E. Cremmer, I. V. Lavrinenko, H. Lu, C. N. Pope, K. S. Stelle and T. A. Tran, "Euclidean-signature supergravities, dualities and instantons," Nucl. Phys. B534, 40 (1998) hep-th/9803259.

[24] S. Rey, "The Confining Phase of Superstrings and Axionic Strings," Phys. Rev. D43 (1991) 526.

[25] E. Cremmer, B. Julia and J. Scherk, "Supergravity theory in 11 dimensions," Phys. Lett. B76 (1978) 409.

[26] A. C. Cadavid, A. Ceresole, R. D'Auria and S. Ferrara, "Eleven-dimensional supergravity compactified on Calabi-Yau threefolds," Phys. Lett. B357 (1995) 76 hepth/9506144.

[27] A. Lukas, B. A. Ovrut, K. S. Stelle and D. Waldram, "Heterotic M-theory in five dimensions," Nucl. Phys. B552 (1999) 246 [hep-th/9806051].

[28] S. Coleman and K. Lee, "Wormholes Made Without Massless Matter Fields," Nucl. Phys. B329 (1990) 387.

[29] S. B. Giddings and A. Strominger, "String Wormholes," Phys. Lett. B230 (1989) 46.

[30] P. van Nieuwenhuizen and A. Waldron, "On Euclidean spinors and Wick rotations," Phys. Lett. B389, 29 (1996) hep-th/9608174.

[31] J. M. Izquierdo, N. D. Lambert, G. Papadopoulos and P. K. Townsend, "Dyonic Membranes," Nucl. Phys. B460 (1996) 560 hep-th/9508177.

[32] M. B. Green, N. D. Lambert, G. Papadopoulos and P. K. Townsend, "Dyonic p-branes from self-dual (p+1)-branes," Phys. Lett. B384 (1996) 86 hep-th/9605146.

[33] D. Sorokin and P. K. Townsend, "M-theory superalgebra from the M-5-brane," Phys. Lett. B412 (1997) 265 hep-th/9708003].

[34] I. Bandos, K. Lechner, A. Nurmagambetov, P. Pasti, D. Sorokin and M. Tonin, "Covariant action for the super-five-brane of M-theory," Phys. Rev. Lett. 78 (1997) 4332 hep-th/9701149.

[35] M. Aganagic, J. Park, C. Popescu and J. H. Schwarz, "World-volume action of the M-theory five-brane," Nucl. Phys. B496 (1997) 191 hep-th/9701166].

[36] O. Barwald, N. D. Lambert and P. C. West, "A calibration bound for the M-theory fivebrane," Phys. Lett. B463 (1999) 33 [hep-th/9907170].

[37] D. Lust and A. Miemiec, "Supersymmetric M5-branes with H-field," Phys. Lett. B476 (2000) 395 hep-th/9912065.

[38] M. Marino, R. Minasian, G. Moore and A. Strominger, "Nonlinear instantons from supersymmetric p-branes," JHEP 0001 (2000) 005 [hep-th/9911206]. 